Gene, 106 (1991) 97-101

(c) 1991 Elsevier Science Publishers B.V. All rights reserved. 0378-1119/91/\$03.50

GENE 06066

\title{
Plasmids for recombination-based screening
}

(DNA sequencing; Escherichia coli; multiplexing; R6K, recombinant DNA; supF; suppressor)

\author{
Gordon D. Stewart, Michael A. Hauser, Harold Kang, Damien P. McCann, Małgorzata M. Osemlak, David M. Kurnit and \\ Andrzej J. Hanzlik
}

Departments of Pediatrics and Human Genetics, Howard Hughes Medical Institute, University of Michigan Medical Center, Ann Arbor. MI 48109-0650 (U.S.A.)

Received by J.L. Slightom: 6 June 1991

Accepted: 1 July 1991

Received at publishers: 30 July 1991

\section{SUMMARY}

To facilitate recombination-based screening, we constructed the ColE1-based plasmid, $\pi \mathrm{G} 4$, that confers chloramphenicol resistance, contains a polylinker with multiple unique restriction enzyme recognition sequences, and contains the genetic marker, supF. To facilitate rccombination-based screening followed by rapid DNA sequencing, we inserted the selectable marker, supF, into each of 20 high-copy-number (hen) pUC-derived NoC plasmids that were designed for multiplex DNA sequencing. To facilitate recombination-based screening of common cDNA libraries that often contain ColE1 sequences, we constructed a supF-carrying plasmid whose replication was driven from an R6K replicon that does not share sequence homology with ColE1. Furthermore, we incorporated a useful polylinker and increased the copy number of this plasmid to create the 4.4-kb hen plasmid, pMAD1. Thus, these plasmids allow: (I) background-free transformation of cells by a supF plasmid carrying an antibiotic-resistance marker; (2) simultaneous performance of the recombination-based assay and DNA sequencing; and (3) screening bacteriophage cDNA libraries that contain ColE1 sequences by recombination with a supF plasmid that is not homologous to ColE1 derivatives.

\section{INTRODUCTION}

Screening bacteriophage libraries by recombination is a rapid technique that promises to play a significant role in

Correspondence to: Dr. D.M. Kurnit, Howard Hughes Medical Institute, University of Michigan Medical Center, 1150 W. Medical Center Dr., Ann Arbor, MI $48109-0650$ (U.S.A.);

Tel. (313)747-4747; Fax (313)936-9353.

Abbreviations: am, amber stop codon; Ap, ampicillin; bp, base pair(s); cDNA, DNA complementary to RNA; Cm, chloramphenicol; hen, high copy number; IPTG, isopropyl- $\beta$-D-thiogalactopyranoside; $\mathrm{kb}$, kilubase(s) or $1000 \mathrm{bp}$; Km, kanamycin; LB, Luria-Bertani (broth); nt, nucleotide(s); oligo, oligodeoxyribonucleotide; pfu, plaque-forming unit(s); ${ }^{\mathrm{R}}$, resistance/resistant; sup $F$, gene encoding mutant tyrosine tRNA that translates an am; Tc, tetracycline; wt, wild type; $\mathrm{XGal}$, 5 -bromo-4-chloro-3-indolyl- $\beta$-D-galactopyranoside; [ ], denotes plasmidcarrier state. the deciphering of complex genomes (Seed, 1983; Kurnit and Seed, 1990). To facilitate this technique, we constructed three types of plasmids. First, we constructed a supF ColE1 plasmid vector that conferred $\mathrm{Cm}$ resistance directly. This plasmid, which has multiple unique cloning sites in the polylinker, avoids the use of an ancillary plasmid to select for $s u p F$. Second, the advent of multiplex sequencing processes establishes an cfficient design for rapid DNA sequencing (Church and Kieffer-Higgins, 1988). We took advantage of this design to construct sup $F$-containing plasmids that would satisfy both multiplex sequencing and recombination-based assay requirements. Third, screening bacteriophage libraries by recombination requires that the libraries are free of the vector sequences used for screening. Because of the presence of ColE1 sequences in $\lambda \mathrm{gt} 11$ (Young and Davis, 1983), $\lambda$ gt10 (Huynh et al., 1985) and phage $\lambda$ libraries constructed from YAC (Burke et al., 
1987) recombinants, background-free recombination-based screening of these libraries requires the construction of a plasmid that carries sup $F$ but lacks sequence overlap with ColE1. We describe herein the elaboration of such a plasmid based on the $\mathrm{R} 6 \mathrm{~K}$ replicon.

\section{EXPERIMENTAL AND DISCUSSION}

\section{(a) Creation of a supF plasmid that confers antibiotic resis- tance}

To ensure the small size of supF plasmids, originally no antibiotic-resistance marker was placed directly on the plasmids; rather the supF marker was selected by requiring suppression of am mutations in antibiotic-resistance encoding genes on another plasmid, p3 $\left(\mathrm{Km}^{\mathrm{R}} \mathrm{Ap}^{\mathrm{R}} \mathrm{am}\right.$ $T_{c^{R}}$ anI ; Seed, 1983). While this was beneficial when very small supF plasmids were required, this strategy was plagued by the occurrence of bacterial chromosome suppressors. Although the frequency of occurrence of such chromosomal suppressors is low, the frequency of recombinant plasmid construction is often low enough or absent so that the cells that grow after selection for supF have the chromosomal, rather than the desired plasmid, suppressor tRNA.

To circumvent this difficulty, we cloned the $\mathrm{Cm}^{\mathrm{R}}$ marker directly onto a plasmid that expresses $\operatorname{supF}$. To accomplish this, a HaeII fragment expressing $\mathrm{Cm}^{\mathrm{R}}$ was taken from pACYC184 (Chang and Cohen, 1978). This fragment was blunt-ended using mung bean nuclease and cloned into the blunt-ended SmaI site in the polylinker of $\pi$ AN13 (Lutz et al., 1987), yielding $\pi$ FLEE6. This vector has two EcoRI sites, one in the polylinker and one in the $\mathrm{Cm}^{\mathrm{R}}$ gene. To eliminate the $E c o$ RI site in the $\mathrm{Cm}^{\mathrm{R}}$ gene of $\pi$ FLEE6, we substituted the 339-bp DraI fragment of this gene from pMLC28 (gift of B. Seed; Burmeister and Lehrach, 1988). Plasmid pMLC28 has this Eco RI site removed by directed mutagenesis of a single bp but still expresses $\mathrm{Cm}^{\mathrm{R}}$. Removal of the EcoRI site in the $\mathrm{Cm}^{\mathrm{R}}$ gene of the resultant plasmid, $\pi \mathrm{G} 13$, was confirmed by restriction digestion and DNA sequencing (data not shown). A synthetic oligo was inserted into the HindIII site in the polylinker of $\pi \mathrm{G} 13$ to construct $\pi \mathrm{G} 2-5$. This oligo furnished NotI and EcoRI sites and recreated the unique HindIII site. The EcoRI site on the other side of the $\mathrm{Cm}^{\mathrm{R}}$ element was removed by digesting with $X m n I+S a c$ I, blunt-ending the Sac I overhang with T4 DNA polymerase, and ligating the blunt-ended fragment.
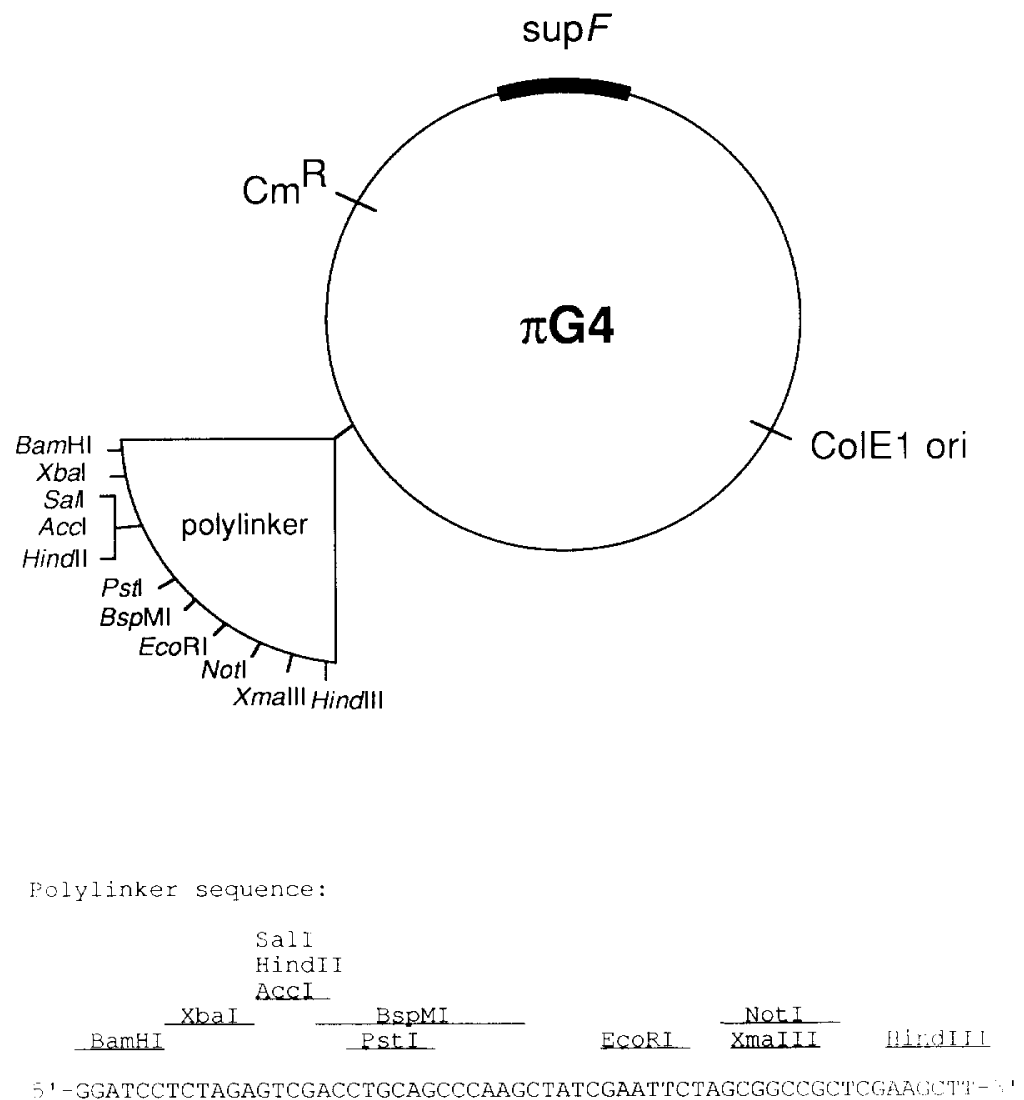

Fig. 1. The organization of $\pi \mathrm{G} 4$. This vector was constructed starting from $\pi \mathrm{AN} 13$ (Lutz et al., 1987). Plasmid $\pi \mathrm{G} 4$ has the following features: a ColE1 origin of replication, a $\mathrm{Cm}^{\mathrm{R}}$ element, a supF gene and a polylinker. The sequence of the polylinker is given. 


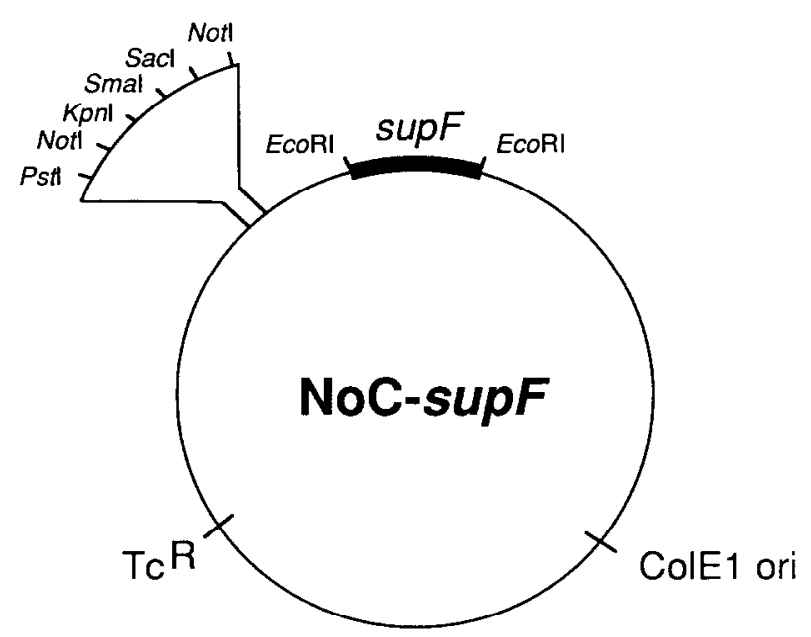

Fig. 2. The organization of NoC-supF vectors. These vectors were constructed starting from the NoC plasmids described by Church and Kieffer-Higgins (1988). In each case, the plasmid was opened at the unique $E c o$ RI site and a 203-bp $E c o$ RI fragment from pGFY $\pi \mathrm{VX}$ (section b) spanning the $\sup F$ gene was inserted.

This engendered the loss of 10 bp including the concomitant destruction of EcoRI, $X m n I$ and SacI recognition sites. Sequencing was performed to ensure that all the changes made to construct $\pi \mathrm{G} 4$ were done correctly. The unique cloning sites in the polylinker are for $A c c \mathrm{I}, B a m \mathrm{HI}, B s p \mathrm{MI}$, EcoRI, HindII, HindIII, NotI, PstI, SalI, XbaI and XmaIII (Fig. 1).

\section{(b) Insertion of $\operatorname{supF}$ into plasmids designed for multiplex sequencing}

Rapid multiplex sequencing demanded the creation of hen plasmids with cloning sites surrounded by different oligos that allowed sequential multiplex sequencing. To meet this need, Church and Kieffer-Higgins (1988) elaborated the NoC family of plasmids which abuts the cloning site of pUC-based hen plasmids with synthetic oligos that permit primed sequencing analyses. We inserted sup $F$ into the EcoRI site of each of the 20 distinct NoC plasmids (Fig. 2). The supF gene was derived from $\mathrm{pGFY} \pi \mathrm{VX}$, constructed by inserting a Pst I fragment encompassing $\pi \mathrm{VX}$ (Sced, 1983) into the PstI site of pGFY218 (Amann et al., 1983). This construct grew better than the original $\pi \mathrm{VX}$ plasmid and was therefore used as the source for supF. An
Eco RI digest of pGFY $\pi \mathrm{VX}$ yielded a 203-bp fragment spanning supF that was ligated into the $E c o$ RI site of each of the NoC plasmids (Church and Kieffer-Higgins, 1988). Twenty ligations were done separately to clone supF into each of the 20 distinct NoC plasmids. The ligations were

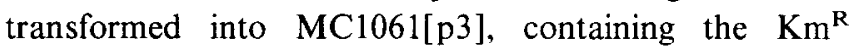
$\mathrm{Ap}^{\mathrm{R}}$ am $\mathrm{Tc}^{\mathrm{R}}$ am plasmid, $\mathrm{p} 3$ (Seed, 1983). The presence of supF was then ascertained by a $\mathrm{Km}^{\mathrm{R}} \mathrm{Ap}^{\mathrm{R}} \mathrm{Tc}^{\mathrm{R}}$ phenotype (Seed, 1983). In addition, each cloning product was checked for functional sup $F$ by the ability of the product to support plating of $\lambda$ phages carrying am mutations in the $\mathrm{A}$ and B genes (Kurnit and Seed, 1990). The result is a series of NoC-supF plasmids that permit both multiplex sequencing and recombination-based screening. The viability of these strains demonstrates that presence of a hen of supF is tolerated.

\section{(c) Insertion of supF into an R6K replicon}

For recombination-based screening (Seed, 1983; Kurnit and Seed, 1990), there can be no shared nt sequence identity between the screening plasmid and the library to be screened which would result in false positives. Unfortunately, $\lambda$ gt 11 libraries (Young and Davis, 1983) require the presence of the ColEl plasmid, pMC9, to suppress expression of the lac promoter in $\lambda \mathrm{gt} 11$. Recombination between phages and this plasmid mediated by shared lac sequences (at a frequency of approx. $10^{-4}$ ) results in the integration of ColE1 (pMC9) sequences into the phages. This prevents background-free recombination-based screening of these libraries with plasmids that contain the ColE1 origin. Although $\lambda \mathrm{gt} 10$ libraries should not suffer this problem theoretically, we have found that many $\lambda \mathrm{gt} 10$ libraries do contain ColE1-derived sequences (Jankowski et al., 1990), indicating that these libraries have been passaged on the strains intended for $\lambda \mathrm{gt} 11$ or that accidental contamination has occurred. Regardless, ColE1-derived replicons cannot be used for recombination-based screening of these $\lambda \mathrm{gt} 10$ cDNA libraries. Vectors carrying YAC DNA contain ColEl sequences that are present in the YAC vehicle (Burke et al., 1987). This prevents background-free recombination-based screening of libraries constructed from YAC vehicles with a sequence inserted in a ColEl origin plasmid.

\section{TABLE I}

Sequences of oligos used herein to construct pMAD1

5'-GTTTCGGACTTTTGAAAG-3'; (end of sup $F$ without polylinker attached); (18 bp)

5'-AATTGTTTCGGACTTTTGAA-3'; mutation underlined; (end of supF without polylinker attached); (20 bp)

5'-AATTGGATCCAGCTGCAGCGGCCGCGGAGGCCGTCGACGCGTGAATTCTTTCTCAACGTAACACTTTAC-3'; mutation underlined; (end of supF with polylinker altached); (69 bp)

5'-GGATCCAGCTGCAGCGGCCGCGGAGGCCGTCGACGCGTGAATTCTTTCTCAACGTAACACTTTAC-3'; (end of supF with polylinker attached); (65 bp) 

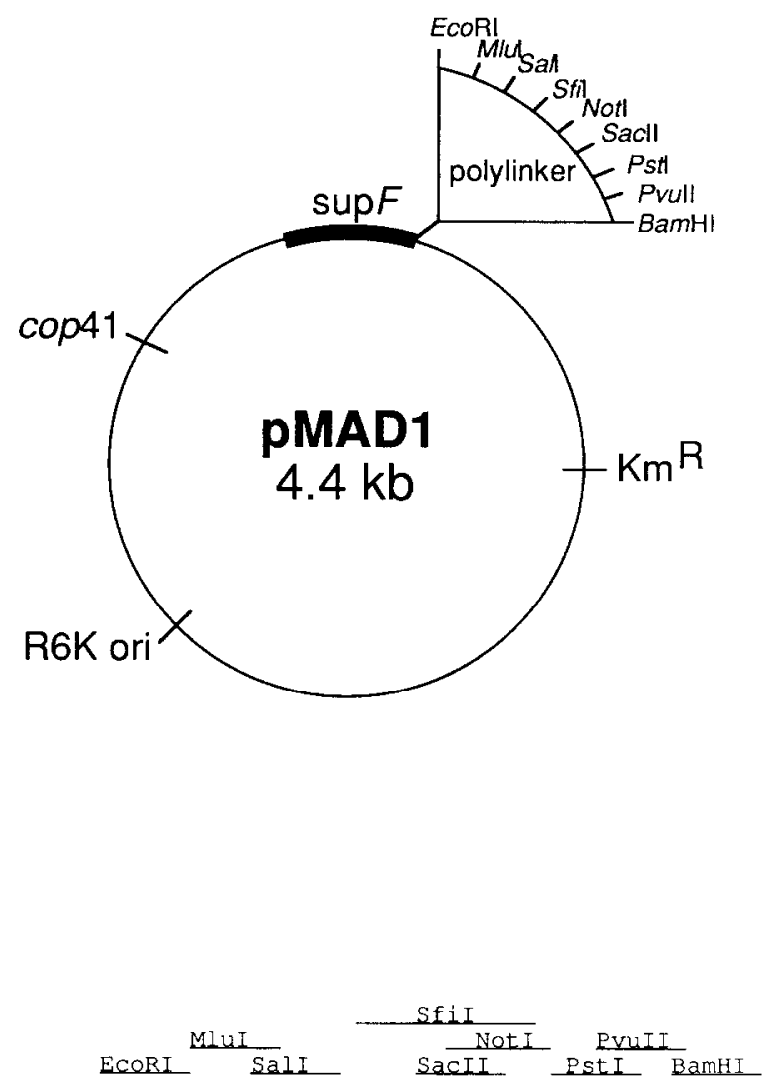

CTCTTACCTTGAGAAAGAATTCACGCGTCGACGGCCTCCGCGGCCGCTGCAGCTGGATCCAATTCCGGAT

$3^{\prime}$ end of supF polylinker

Fig. 3. The organization of pMAD1. This plasmid was constructed starting from the R6K-derived element, pcos2EMBL (Poustka et al., 1984). The large $E c o$ RI fragment of pcos2EMBL was ligated to a PCR segment that spanned the sup $F$ gene, destroyed these $E c o$ RI sites and generated a polylinker whose sequence is given. This intermediate was termed pAD1. The small HgiAI-BanI fragment of pAD1 was replaced by the small $H g i A I-B a n I$ fragment of pGN7-cop 41 (provided by M. Inuzuka and P. Wada; Inuzuka and Wada, 1985) to create the hen plasmid, pMAD1. The overall structure and polylinker of this plasmid are given.

To eliminate this background, we cloned supF into an R6K plasmid replicon which is not homologous to ColE1 (Kolter and Helinski, 1978; Poustka et al., 1984). Again, the EcoRI fragment encompassing the supF gene in pGFY $\pi \mathrm{VX}$ was the source of supF for the construction of this R6K-derived vector. Two PCR amplifications (Saiki et al., 1985) were performed using the oligo primers in Table I to construct equivalent length fragments that spanned the supF gene of pGFY $\pi \mathrm{VX}$ and that were offset by $4 \mathrm{bp}$. In addition to amplifying the supF gene, the oligo primers used for these PCR reactions created a polylinker containing sites for BamHI, EcoRI, MluI, NotI, PstI, PvuII, SacII, SalI and SfiI (Fig. 3). When the products of these two PCR amplifications were denatured and annealed (Jones et al., 1990) the resultant mixture contained both blunt-ended and staggered-ended molecules. By design, the latter had 5'-AATT-3' overhangs compatible with EcoRI overhangs. Further, the oligos used to prime the PCR (Table I) were constructed so that a 1-bp mismatch resulted in the destruction of intact $E c o$ RI sites at each end by mutation of the $3^{\prime}$-most nt of the Eco RI recognition sequence from $\mathrm{C}$ to G. However, the continued presence of 5'-AATT-3' ensured that each overhang would still ligate with an EcoRI overhang. These sup $F$-polylinker fragments generated by PCR lacked 5' -phosphates by virtue of their mode of synthesis. Thus, a large excess of these fragments could be added without ligation occurring among them. An EcoRI digest of pcos2EMBL (Poustka et al., 1984) resulted in the liberation of the $\mathrm{R} 6 \mathrm{~K}$ replicon and $\mathrm{a} \mathrm{Km}^{\mathrm{R}}$ element together lacking identity to pBR322 or phage $\lambda$ (Poustka et al., 1984). This $E c o$ RI fragment was ligated to the sticky-ended $s u p F$ fragment generated during the two PCRs followed by denaturation and annealing. After transformation into the $\mathrm{Su}^{0}$ lacZam host, LG75 (Guarente et al., 1980), $\mathrm{Km}^{\mathbf{R}} \mathrm{Tc}^{\mathrm{S}}$ colonies that were blue in the presence of IPTG and XGal had the desired $\mathrm{Km}^{\mathrm{R}} \mathrm{Tc}^{\mathrm{S}}$ sup $F$ genotype with the designed polylinker. The plasmid in one such colony was termed pAD1.

Increasing the copy number of pAD 1 required insertion of a cop 41 mutation (Inuzuka and Wada, 1985). This single 
bp change was shown previously to increase the copy number of R6K from approx. 20 to approx. 100 (Inuzuka and Wada, 1985). The cop 41 mutation was isolated on a BanI-HgiAI restriction fragment from pGN7 cop 41 (Inuzuka and Wada, 1985). This fragment was ligated to the large BanI-HgiAI fragment of pAD1. Following transformation into the lac $Z \mathrm{am} \mathrm{Su}{ }^{0}$ host. LG75, the $\mathrm{Km}^{\mathrm{R}}$ supF plasmid, pMAD1, was isolated with this hen cop4l mutation.

To demonstrate that the $\operatorname{cop} 41$ mutation was present, we distinguished the wt sequence from the cop 41 sequence by the presence of an $R s a I$ site engendered by the normal sequence but not by the mutant. The region in question was amplified by PCR using two oligos, one of which abutted the cop 41 mutation and the other was in $\operatorname{supF}$. RsaI digestion of this PCR fragment yielded a 220-bp fragment if the wt sequence was present and a 240-bp fragment if the mutant cop 41 sequence was present. This analysis confirmed that pMAD1 contained the desired cop 41 mutation.

To underscore that pMADI lacks identity to ColEl sequences, we showed by recombination (Seed, 1983; Kurnit and Seed, 1990) that this plasmid does not recombine with ColE1 nt sequences in $\lambda$ phages. This lack of identity is consistent with the known sequence of R6K and with the finding that R6K-based replicons do not recombine with cosmids carrying ColEl sequences (Poustka et al., 1984). In summary, pMAD1 is a 4.4-kb hon R6K-derived plasmid that confers $\mathrm{Km}^{\mathrm{R}}$ and $\sup F$, has a polylinker with multiple cloning sites and is not homologous to ColE1 plasmids (Fig. 3). This plasmid can be used to screen libraries fabricated in phage $\lambda$ regardless of whether the ColE1 replicon is present therein. To illustrate this point, we have used this plasmid to study the transcription pattern of more than 500 genomic sequences (M.A.H., M.M.O., D.M.K. and A.J.H., unpublished).

The result of these constructions is that the applicability of the recombination-based assay is broadened. In particular, the last application permits us to screen a wide variety of cDNA libraries constructed in vectors that have ColE1 sequences. This increases the number and kind of libraries that are amenable to the recombination-based approach.

\section{ACKNOWLEDGEMENTS}

We thank Brian Seed for providing pMLC28, Hans Lehrach for providing pcos2EMBL, and George Church for providing the NoC plasmids. Frederick Lee constructed the $\pi$ FLEE6 plasmid. M.A.H., M.M.O. and A.J.H. are Associates and D.M.K. is an Investigator of the Howard Hughes Medical Institute. Supported also by a grant from the Alzheimer's Disease and Related Disorders Association to G.D.S.

\section{REFERENCFS}

Amann, E., Brosius, J. and Ptashne, M.: Vectors bearing a hybrid trp-lac promoter useful for regulated expression of cloned genes in Escherichia coli. Gene 25 (1983) 167-178.

Burke, D.T., Carle, G.F. and Olson, M.V.: Cloning of large segments of exogenous DNA into yeast by means of artificial chromosome vectors. Science 236 (1987) 806-812.

Burmeister, M. and Lehrach, H.: Jekyll, a family of phage-plasmid shuttle vectors. Gene 73 (1988) 245-250.

Chang, A.C.Y. and Cohen, S.N.: Construction and characterization of amplifiable multicopy DNA cloning vehicles derived from the P15A cryptic miniplasmid. J. Bacteriol. 134 (1978) 1141-1156.

Church, G.M. and Kieffer-Higgins, S.: Multiplex DNA sequencing. Science 240 (1988) 185-188.

Guarente, L., Lauer, G., Roberts, T.M. and Ptashne, M.: Improved methods for maximizing expression of a cloned gene: a bacterium that synthesizes rabbit $\beta$-globin. Cell 20 (1980) 543-553.

Inuzuka, M. and Wada, Y.: A single amino acid mutation in the initiation protein is responsible for the DNA overproduction phenotype of copy number mutants of plasmid R6K. EMBO J. 4 (1985) 2301-2307.

Jankowski, S., Stewart, G.D., Buraczynska, M., Galt, J., Van Keuren, M. and Kurnit, D.M.: Molecular approaches to trisomy 21. In: Epstein, C. and Patterson, D. (Eds.), 21st Chromosome and Down Syndrome, Wiley-Liss, New York, 1990, pp. 79-88.

Jones, D.H., Sakamoto, K., Vorce, R.L. and Howard, B.H.: DNA mutagenesis and recombination. Nature 344 (1990) 793-794.

Kolter, R. and Helinski, D.R.: Construction of plasmid R6K derivatives in vitro: characterization of the $\mathrm{R} 6 \mathrm{~K}$ replication region. Plasmid 1 (1978) 571-580.

Kurnit, D.M. and Seed, B.: Improved genetic selection for screening bacteriophage libraries by homologous recombination in vivo. Proc. Natl. Acad. Sci. USA 87 (1990) 3166-3169.

Lutz, C.T., Hollifield, W.C., Seed, B., Davie, J.M. and Huang, H.V.: Syrinx 2A: an improved $\lambda$ phage vector designed for screening DNA libraries by recombination in vivo. Proc. Natl. Acad. Sci. USA 84 (1987) 4379-4383.

Poustka, A., Rackwitz, H.-R., Frischauf, A., Hohn, B. and Lehrach, H.: Selective isolation of cosmid clones by homologous recombination in Escherichia coli. Proc. Natl. Acad. Sci. USA 81 (1984) 4129-4133.

Saiki, R.K., Scharf, S., Faloona, F., Mullis, K.B., Horn, G., Erlich, H.A. and Arnheim, N.: Enzymatic amplification of $\beta$-globin genomic sequences and restriction site analysis for diagnosis of sickle cell anemia. Science 230 (1985) 1350-1354.

Secd, B.: Purification of genomic scquences from bacteriophage libraries by recombination and selection in vivo. Nucleic Acids Res. 11 (1983) 2427-2445.

Young, R.A. and Davis, R.W.: Efficient isolation of genes by using antibody probes. Proc. Natl. Acad. Sci. USA 80 (1983) 1194-1198. 